\title{
Detecting communicative intent in a computerised test of joint attention
}

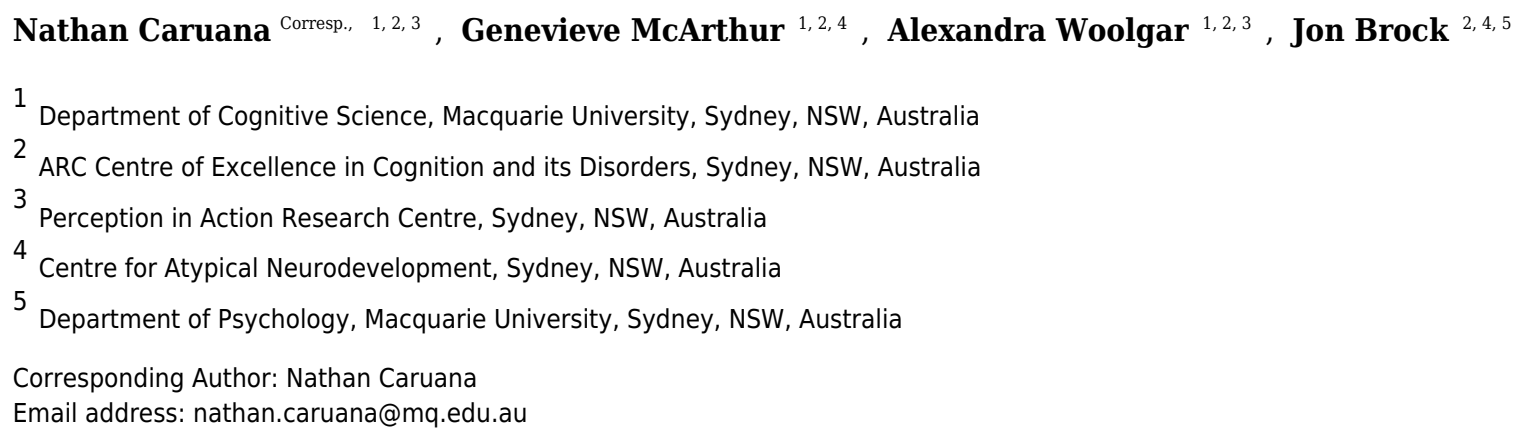

The successful navigation of social interactions depends on a range of cognitive faculties including the ability to achieve joint attention with others to share information and experiences. We investigated the influence that intention monitoring processes have on gaze-following response times during joint attention. We employed a virtual reality task in which 16 healthy adults engaged in a collaborative game with a virtual partner to locate a target in a visual array. In the Search task, the virtual partner was programmed to engage in non-communicative gaze shifts in search of the target, establish eye contact, and then display a communicative gaze shift to guide the participant to the target. In the NoSearch task, the virtual partner simply established eye contact and then made a single communicative gaze shift towards the target (i.e., there were no non-communicative gaze shifts in search of the target). Thus, only the Search task required participants to monitor their partner's communicative intent before responding to joint attention bids. We found that gaze following was significantly slower in the Search task than the NoSearch task. However, the same effect on response times was not observed when participants completed non-social control versions of the Search and NoSearch tasks, in which the avatar's gaze was replaced by arrow cues. These data demonstrate that the intention monitoring processes involved in differentiating communicative and non-communicative gaze shifts during the Search task had a measurable influence on subsequent joint attention behaviour. The empirical and methodological implications of these findings for the fields of autism and social neuroscience will be discussed. 


\section{Corresponding Author:}

15 Dr. Nathan Caruana

Email: nathan.caruana@mq.edu.au 


\section{ABSTRACT}

19 The successful navigation of social interactions depends on a range of cognitive faculties -

20 including the ability to achieve joint attention with others to share information and experiences.

21 We investigated the influence that intention monitoring processes have on gaze-following

22 response times during joint attention. We employed a virtual reality task in which 16 healthy

23 adults engaged in a collaborative game with a virtual partner to locate a target in a visual array.

24 In the Search task, the virtual partner was programmed to engage in non-communicative gaze

25 shifts in search of the target, establish eye contact, and then display a communicative gaze shift

26 to guide the participant to the target. In the NoSearch task, the virtual partner simply established

27 eye contact and then made a single communicative gaze shift towards the target (i.e., there were

28 no non-communicative gaze shifts in search of the target). Thus, only the Search task required

29 participants to monitor their partner's communicative intent before responding to joint attention

30 bids. We found that gaze following was significantly slower in the Search task than the

31 NoSearch task. However, the same effect on response times was not observed when participants

32 completed non-social control versions of the Search and NoSearch tasks, in which the avatar's

33 gaze was replaced by arrow cues. These data demonstrate that the intention monitoring processes

34 involved in differentiating communicative and non-communicative gaze shifts during the Search

35 task had a measurable influence on subsequent joint attention behaviour. The empirical and

36 methodological implications of these findings for the fields of autism and social neuroscience

37 will be discussed.

38 Joint attention is defined as the simultaneous coordination of attention between a social

39 partner and an object or event of interest (Bruner, 1974; 1995). It is an intentional,

40 communicative act. In the prototypical joint attention episode, one person initiates joint attention 
41 (IJA) by pointing, turning their head, or shifting their eye gaze to intentionally guide their social

42 partner to an object or event in the environment. The partner must recognise the intentional

43 nature of this initiating behaviour and respond to that joint attention bid (RJA) by directing their

44 attention to the cued location (Bruinsma, Koegel, \& Koegel, 2004).

The ability to engage in joint attention is considered critical for the normal development of

46 language and for navigating social interactions (Adamson, Bakeman, Deckner, \& Romski, 2009;

47 Charman, 2003; Dawson et al., 2004; Mundy, Sigman, \& Kasari, 1990; Murray et al., 2008;

48 Tomasello, 1995) and its developmental delay is a hallmark of autism spectrum disorders (Lord 49 et al., 2000; Stone, Ousley, \& Littleford, 1997). Yet despite its importance to both typical and

50 atypical development, very little is known about the neurocognitive mechanisms of joint

51 attention. By definition, joint attention involves an interaction between two individuals. The

52 challenge for researchers, therefore, has been to develop paradigms that achieve the ecological

53 validity of a dynamic, interactive, social experience, whilst at the same time maintaining 54 experimental control.

55 In a recent functional magnetic resonance imaging (fMRI) study, Schilbach et al. (2010)

56 investigated the neural correlates of joint attention using a novel virtual reality paradigm. During

57 the scan, participants' eye-movements were recorded as they interacted with an anthropomorphic 58 avatar. They were told that the avatar's gaze was controlled by a confederate outside the scanner 59 also using an eye-tracking device. In fact, the avatar was controlled by a computer algorithm that 60 responded to the participant's own eye-movements. On RJA trials (referred to as OTHER_JA by 61 Schilbach et al., 2010), the avatar looked towards one of three squares positioned around his face, 62 and participants were instructed to respond by looking at the same square. Participants also 63 completed IJA trials in which the roles were reversed. 
64 Similar tasks have been used in other fMRI studies using either gaze-contingent avatars

65 (Oberwelland et al., 2016) or live-video links to a real social partner (Redcay et al., 2012; Saito

66 et al., 2010). Together, these interactive paradigms represent an important step towards an

67 ecologically valid measure of joint attention. There is, however, a potentially important

68 limitation of the tasks used in these studies: in each task, every trial involved a single

69 unambiguously communicative eye-gaze cue. On RJA trials, the participant's partner would

70 make a single eye-movement towards the target location and the participant knew they were

71 required to respond to that isolated cue. This differs from real-life joint attention episodes, which

72 are embedded within complex ongoing social interactions. In real life, responding to a joint

73 attention bid requires that the individual first identifies the intentional nature of their partner's

74 behaviour. That is, they must decide whether or not the cue is one they should follow. We refer

75 to this component of joint attention as "intention monitoring".

76 In a recent $\mathrm{fMRI}$ study, we developed a novel joint attention task to better capture this

77 intention monitoring process (Caruana, Brock \& Woolgar, 2015). Following Schilbach et al.

78 (2010), participants played a cooperative game with an avatar whom they believed to be

79 controlled by a real person (referred to as "Alan"), but was actually controlled by a gaze-

80 contingent algorithm. The participant and avatar were both allotted onscreen houses to search for

81 a burglar (see Figure 1). On IJA trials, the participant found the burglar, made eye contact with

82 the avatar, and then guided the avatar to the burglar by looking back at the house in which the

83 burglar was hiding. On RJA trials, the participant found all of their allotted houses to be empty.

84 Once Alan had finished searching his own houses, he would make eye contact with the

85 participant before guiding them towards the house containing the burglar. 


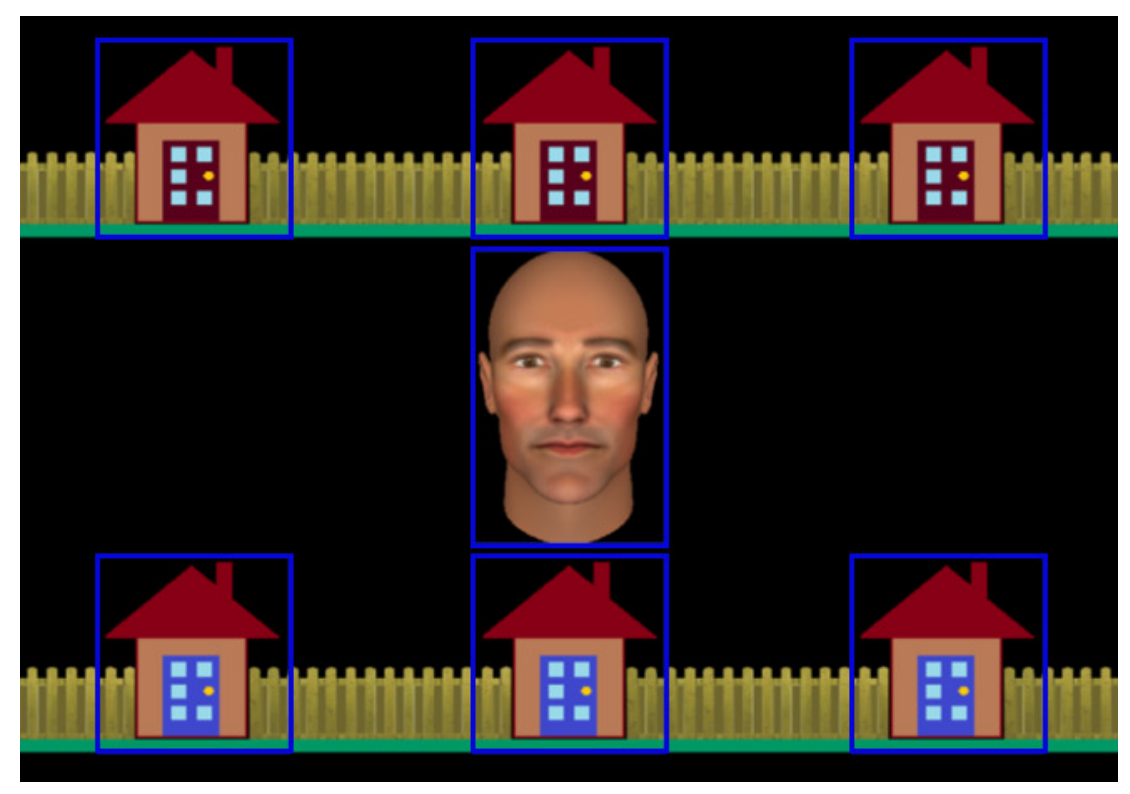

Figure 1. Experimental display showing the central avatar (“Alan”) and the six houses in which the burglar could be hiding. Gaze areas of interest (GAOIs), are represented by blue rectangles, and were not visible to participants.

The critical innovation of this task was the initial search phase. This provided a natural and intuitive context in which participants could determine, on each trial, their role as either the responder or initiator in a joint attention episode (previous studies had provided explicit instructions; e.g., Schilbach et al., 2010; Redcay et al., 2012). More importantly for current

102 purposes, the RJA trials required participants to monitor their partner's communicative

103 intentions. During each trial, the avatar made multiple non-communicative eye-movements as he 104 searched his own houses. The participant had to ignore these eye-movements and respond only 105 to the communicative "initiating saccades" that followed the establishment of eye contact. This is 106 consistent with genuine social interactions in which eye contact is used to signal one's readiness 107 and intention to communicate, particularly in the absence of verbal cues (Cary, 1978). 
We compared the RJA trials in this new paradigm to non-social control trials (referred to as

109

110

111

112

113

114

115

RJAc) in which the eye gaze cue was replaced by a green arrow superimposed over the avatar's

face. Analysis of saccadic reactions times revealed that participants were significantly slower (by approximately $209 \mathrm{~ms}$ ) to respond to the avatar's eye gaze cue than they were to respond to the arrow (Caruana et al., 2015). This effect was surprising - previous studies have shown that gaze cues often engender rapid and reflexive attention shifts (see Frischen, Bayliss, and Tipper, 2007), but that would predict faster rather than slower responses to gaze cues. Nevertheless, we have since replicated this finding in an independent sample of adults and, intriguingly, found that the effect is exaggerated in a group of autistic individuals (Caruana, Stieglitz Ham et al., in press). One explanation for these findings is that they reflect the intention monitoring aspects of RJA. Specifically, participants are slower to respond to eye gaze cues than arrows because it takes time to identify the cue as being an intentional and communicative bid to initiate joint attention. In the control condition, the arrow presents an unambiguous attention cue, and so the participant does not need to decide whether they should respond to it or not. The implication here is that intention monitoring is a cognitively demanding operation that requires time to complete and is manifest in the response times to eye gaze cues.

However, before reaching such a conclusion, it is important to consider a number of alternative explanations. For example, it may be that participants responded faster in the RJAc condition because the large green arrow cue, which extended towards the target location, provided a more salient spatial cue than the avatar's eyes. It is also possible that the mere context of social interaction may influence the way participants approach the task. In particular, when individuals believe they are interacting with an intentional human agent, mirroring and mentalising mechanisms are automatically recruited which exert a top-down effect on the neural 
131 processes governing visual perception or attention (Wykowska, Wiese, Prosser, Müller \&

132 Hamed, 2014).

133 The aim of the current study, therefore, was to test the intention monitoring account more

134 directly by manipulating the intention monitoring component of the RJA task whilst controlling

135 for both the perceptual properties of the stimulus and the social nature of the task. To this end,

136 we tested a new sample of participants using the same task but with one further manipulation. On

137 half the trials, we eliminated the search phase of the task. Thus, on RJA trials, the avatar only

138 made a single eye movement to the target to initiate joint attention, and participants knew

139 unambiguously that they should follow it. The gaze cues in the 'Search' and 'NoSearch' versions

140 of the task were identical and in both cases, participants believed they were interacting with

141 another human. Thus, only the intention monitoring account predicts an effect of task (Search

142 versus NoSearch) on response times. Participants also completed Search and NoSearch versions

143 of the control (RJAc) condition. Because the arrow cue is unambiguous whether or not it is

144 preceded by a search phase, we did not predict any difference in response times. In other words,

145 a condition (Social vs. Control) by task (Search vs. NoSearch) interaction would indicate that

146 response times to joint attention bids are influenced by the intention monitoring processes that

147 precede true RJA behaviours.

Method

\section{Ethical Statement}

150 The study was approved by the Human Research Ethics Committee at Macquarie

151 University (MQ; reference number: 5201200021). Participants received course credit for their

152 time and provided written consent before participating.

\section{Participants}


154 Sixteen right-handed adults with typical development, normal vision, and no history of

155 neurological impairment participated in this study ( 3 female, $M_{\text {age }}=19.92, S D=1.03$ ).

156 Stimuli

157 We employed an interactive paradigm that we had previously used to investigate the 158 neural correlates of RJA and IJA (Caruana, et al., 2015). The stimuli comprised an 159 anthropomorphic avatar face, generated using FaceGen (Singular Inversions, 2008), that 160 subtended 6.5 degrees of visual angle. The avatar's gaze was manipulated so that it could be 161 directed either at the participant or towards one of the six houses that were presented on the 162 screen (see Figure 1). The houses were arranged in two horizontal rows above and below the 163 avatar and each subtended four degrees of visual angle. 


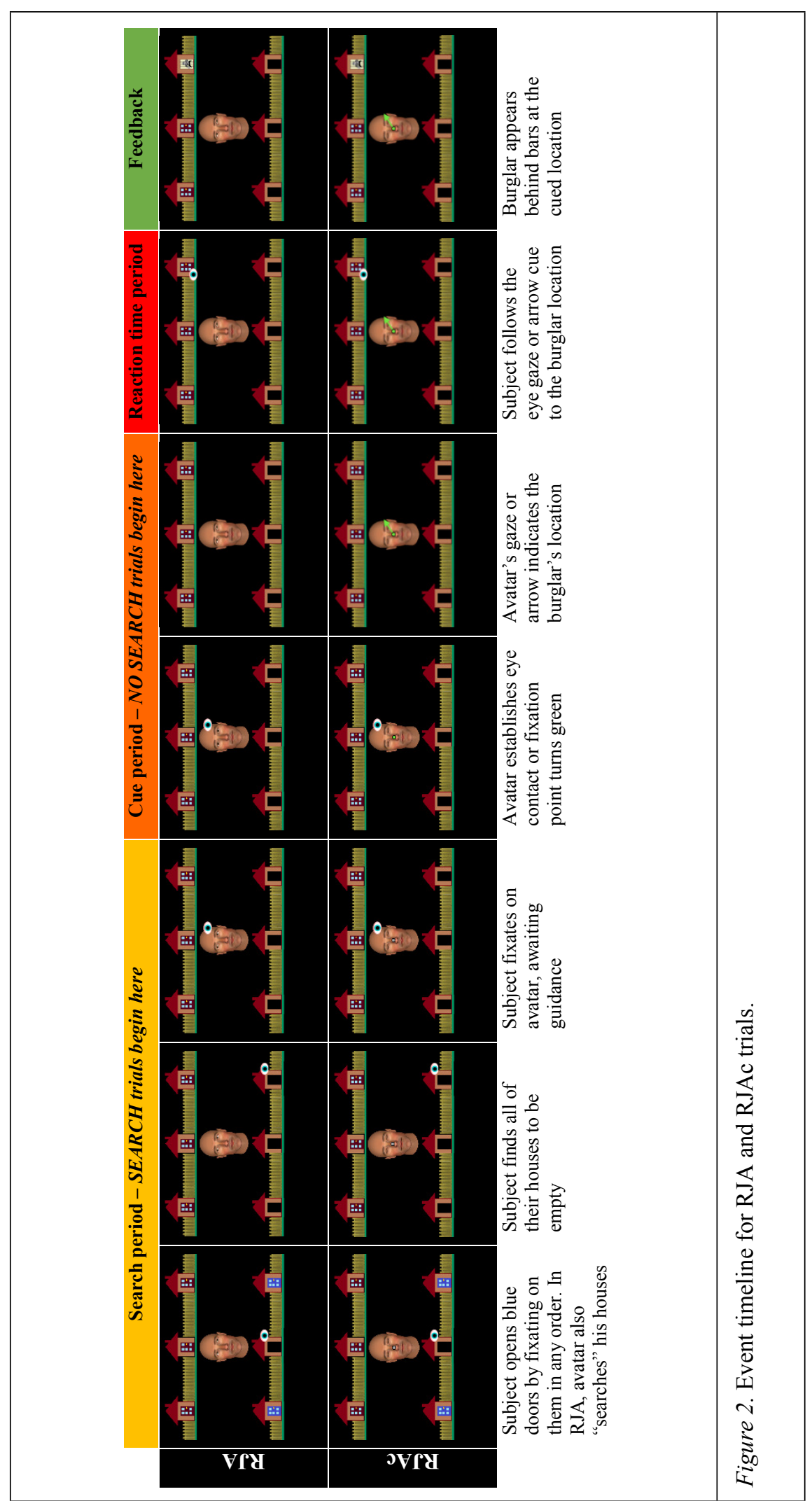


Social Conditions (RJA and IJA). Participants played a cooperative "Catch-the-

166 Burglar" game with an avatar whom they believed was controlled by another person named

167 "Alan" in a nearby eye tracking laboratory using live infrared eye tracking. In reality, a gaze-

168 contingent algorithm controlled the avatar's responsive behaviour (see Caruana et al., 2015 for a

169 detailed description of this algorithm and a video demonstration of the task). The goal of the

170 game was to catch a burglar that was hiding inside one of the six houses presented on the screen.

171 Participants completed two versions of the social conditions (i.e., Search and NoSearch tasks)

172 during separate blocks.

173 Search Task. This task was identical to the "Catch-the-Burglar" task employed in our

174 previous work (e.g., Caruana et al., 2015). Each trial in the Search task began with a "search

175 phase". During this period, participants saw two rows of houses on a computer screen including

176 a row of three blue doors and a row of three red doors. They were instructed to search the row of

177 houses with blue doors while Alan searched the row of houses with red doors. Participants were

178 told that they could not see the contents of Alan's houses and that Alan could not see the

179 contents of their houses. Whoever found the burglar first had to guide the other person to the

180 correct location.

181

Participants searched the houses with blue doors in any order by fixating on them. Once a

182 fixation was detected on a blue door, it opened to reveal either the burglar or an empty house. On

183 some trials, only one or two blue doors were visible, whilst the remaining doors were already

184 open. This introduced some variability in the order with which participants searched their houses 185 that made Alan's random search behaviour appear realistically unpredictable.

Once the participant fixated back on the avatar's face, Alan was programmed to search 0-

1872 more houses and then make eye contact. This provided an interval in which participants could 
188 observe Alan's non-communicative gaze behaviour as he completed his search. The onset

189 latency of each eye movement made by Alan was jittered with a uniform distribution between $190 \quad 500-1000 \mathrm{~ms}$.

191 On RJA trials, participants discovered that all of their allotted houses were empty (Figure

192 2, row 1), indicating that the burglar was hiding in one of Alan's houses. Once the participant

193 fixated back on Alan's face, he searched 0-2 more houses in random order before establishing 194 eye contact with the participant. Alan then initiated joint attention by directing his gaze towards 195 one of his allotted houses. If the participant made a "responding saccade" and fixated the correct 196 location, the burglar was captured.

On IJA trials, the participant found a burglar behind one of the blue doors. They were 198 then required to fixate back on Alan's face, at which point the door would close again to conceal 199 the burglar. Again, Alan was programmed to search 0-2 houses before looking straight at the 200 participant. Once eye contact was established, participants could initiate joint attention by 201 making an "initiating saccade" to fixate on the blue door that concealed the burglar. Alan was 202 programmed to only respond to initiating saccades that followed the establishment of eye 203 contact, and to follow the participant's gaze, irrespective of whether the participant fixated the 204 correct house or not. Whilst performance on IJA trials was not of interest in the current study, the 205 inclusion of this condition created a context for the collaborative search element of the task and 206 allowed direct comparison with our previous studies in which participants alternated between 207 initiating and responding roles.

208 When the participant made a responding or initiating saccade to the correct location, the 209 burglar appeared behind prison bars to indicate that he had been successfully captured (e.g.,

210 Figure 2, column 7). However, the burglar appeared in red at his true location, to indicate that he 
211 had escaped, if participants (1) made a responding or initiating saccade to an incorrect location,

212 (2) took longer than three seconds to make a responding or initiating saccade, or (3) spent more

213 than three seconds looking away from task-relevant stimuli (i.e., Alan and houses). Furthermore,

214 trials were terminated if the participants took longer than three seconds to begin searching their

215 houses at the beginning of the trial. On these trials, red text reading "Failed Search" appeared on

216 the screen to provide feedback.

217 NoSearch Task. This version of the task was identical to the Search task except that the

218 search phase in each trial was removed. In IJA trials, all but one house was visibly empty (i.e.,

219 the door was open and no burglar was present), and participants were instructed that if they saw a

220 blue door in their allotted row of houses that the burglar would be "hiding" behind it. In RJA

221 trials, all of the houses were visibly empty. For both IJA and RJA trials, Alan's eyes would be

222 closed at the beginning of the trial, and then open after 500-1000 ms (jittered with a uniform

223 distribution) so that he was looking at the participant. Alan would then wait to be guided on IJA

224 trials. On RJA trials, Alan shifted his gaze to guide the participant after a further 500-1000 ms,

225 provided that eye contact had been maintained. Thus, in both the Search and NoSearch tasks,

226 Alan made eye contact with the participant before guiding them to the burglar on RJA trials.

227 Therefore, the perceptual properties of the gaze cue itself were identical between tasks, but the

228 NoSearch task removed the requirement to use the eye contact cue to identify communicative 229 gaze shifts.

230 Control Conditions (RJAc and IJAc). For each of the social conditions in both versions

231 of the task, we employed a control condition that was closely matched on non-social task

232 demands (e.g., attentional orienting, oculomotor control). In these conditions (RJAc and IJAc),

233 participants were told that they would play the game without Alan, whose eyes remained closed 
234 during the trial. Participants were told that the stimuli presented on the computer screen in these

235 trials were controlled by a computer algorithm. In the Search task, a grey fixation point was

236 presented over the avatar's nose until the participant completed their search and fixated upon it.

237 After a short delay, the fixation point turned green (analogous to the avatar making eye contact).

238 From this point onwards, the Search and NoSearch tasks were identical. On IJAc trials, the green

239 fixation point was the cue to saccade towards the burglar location. On RJAc trials a green arrow

240 subtending three degrees of visual angle cued the burglar's location (analogous to Alan's guiding

241 gaze; see Caruana et al., 2015 for a video with example trials from each condition).

\section{Procedure}

Joint attention task. The experiment was presented using Experiment Builder 1.10.165

244 (SR Research, 2004). Participants completed four blocks, each comprising 108 trials: two blocks

245 involved the Search task, and another two blocks involved the NoSearch task. Search and

246 NoSearch block pairs were presented consecutively, however their order was counterbalanced

247 across participants. Within each pair of Search and NoSearch blocks, one block required the

248 participant to monitor the upper row of houses, and the other required them to monitor the lower 249 row of houses.

250

Each block comprised 27 trials from each condition (i.e., RJA, RJAc, IJA, IJAc). Social

251 (RJA, IJA) and control (RJAc, IJAc) trials were presented in clusters of six trials throughout

252 each block. Each cluster began with a cue lasting $1000 \mathrm{~ms}$ that was presented over the avatar

253 stimulus and read "Together" for a social cluster and "Alone" for a control cluster. Trial order

254 randomisation was constrained to ensure that the location of the burglar, the location of blue

255 doors, and the number of gaze shifts made by the avatar were matched within each block and

256 condition. 
After playing the interactive game, and consistent with our previous studies employing

258

259

260

261

262

263

264

265

266

267

268

269

270

271

272

273

274

275

276

277 278 period).

\section{Statistical Analyses}

\section{Scores}

this paradigm, a post-experimental interview was conducted in which participants were asked to rate their subjective experience during the task (cf. Caruana et al., 2015; Caruana, Ham et al., in pres). Full details on the assessment of subjective experiences and relevant findings are provided in Supplementary Material 2.

Eye tracking. Eye-movements from the right eye only were recorded with a sampling rate of $500 \mathrm{~Hz}$ using a desktop-mounted EyeLink 1000 Remote Eye-Tracking System (SR Research Ltd., Ontario, Canada). Head movements were stabilised using a chinrest. We conducted an eye tracking calibration using a 9-point sequence at the beginning of each block. Seven gaze areas of interest (GAOIs) over the houses and avatar stimulus were used by our gazecontingent algorithm (see Caruana et al., 2015 for details). A recalibration was conducted if the participant made consecutive fixations on the borders or outside the GAOIs. Trials requiring a recalibration were excluded from all analyses.

Accuracy. We calculated the proportion of trials where the participant succeeded in catching the burglar in each condition (i.e., RJA and RJAc) for each task separately (i.e., Search and NoSearch). We excluded from the accuracy analysis any trials that required a recalibration or (in the Search task) were failed due to an error during the search phase.

Saccadic Reaction Times. For correct trials, we measured the latency (in ms) between the presentation of the gaze cue (for RJA trials) or the arrow cue (for RJAc trails), and the onset of the participant's responding saccade towards the burglar location (see Figure 2, Reaction time 
280 Saccadic reaction times were analysed via repeated measures Analysis of Variance 281 (ANOVA) using the ezANOVA (ez) package in R (Lawrence, 2013), reporting the generalised

282 eta squared $\left(\eta_{G}^{2}\right)$ measure of effect size. Significant task*condition interactions effects were 283 followed-up with Welch's two sample unequal variances t-tests (Welch, 1947). As in our 284 previous studies, we report analyses of the mean reaction time, having excluded trials with 285 reaction times less than $150 \mathrm{~ms}$ as these are typically considered to be anticipatory responses. 286 Trials timed out after $3000 \mathrm{~ms}$ providing a natural upper limit to reaction times. Full syntax and 287 output for this analysis can be found in the Rmarkdown document (Supplementary Material 1). 288 The RMarkdown also provides complementary ANOVAs of the mean and median of the 289 untrimmed data, as well as a mixed random effects analysis using the lme4 R package (Bates, 290 2005). The results of all analyses are consistent in terms of the predicted interaction between task 291 and condition. A significance criterion of $p<0.05$ was used for all analyses. 

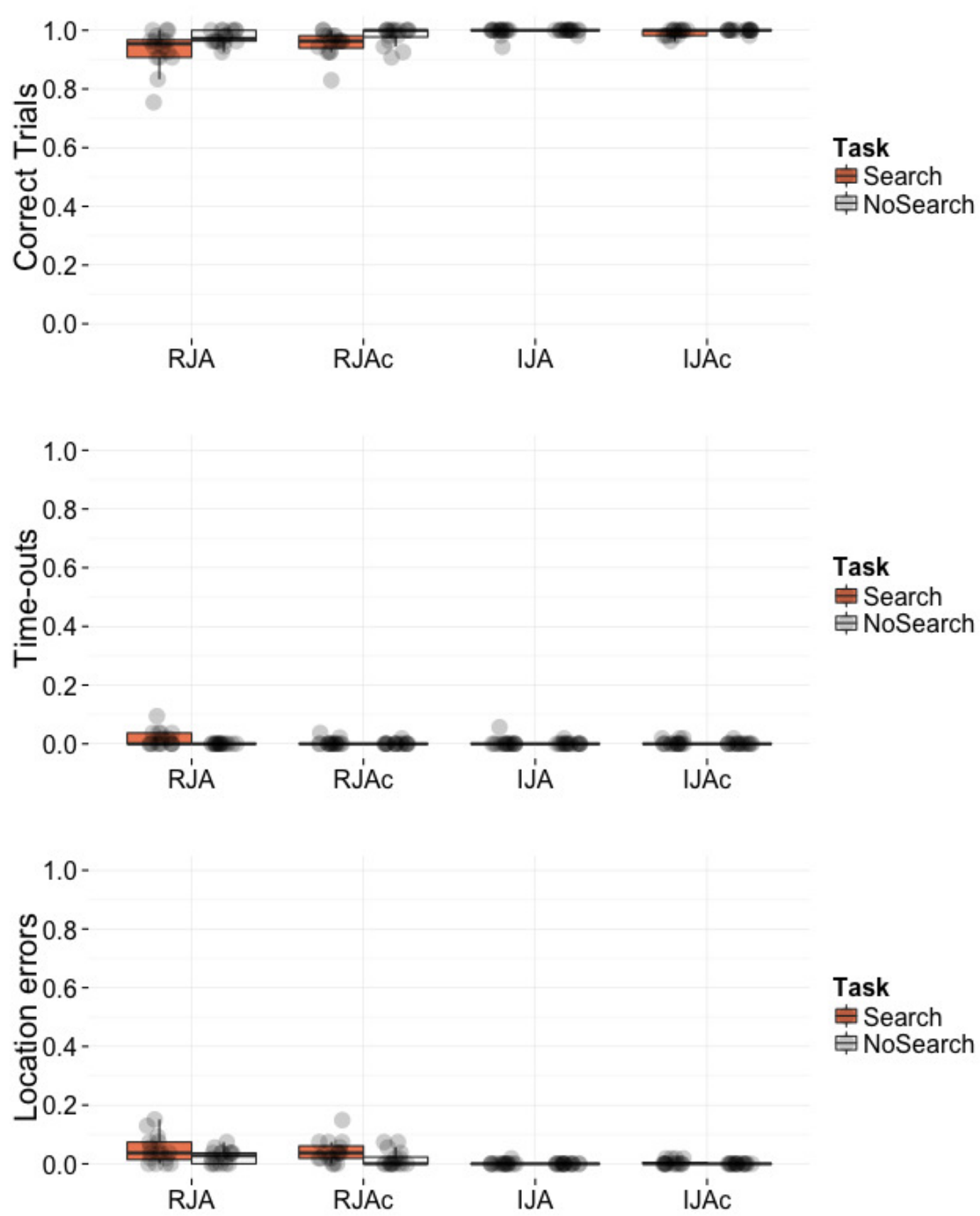

295 Figure 3. Box plots displaying the proportion of correct trials, proportion of time-out errors, and proportion of 296 location errors. Data points represent individual participant means. 
300 trials successfully completed across all conditions. Of the small number of errors made, the

301 majority were Location Errors in the RJA and RJAc conditions, whereby participants looked first

302 to an incorrect location (house) rather than following the avatar's gaze to the burglar location.

303 Given the low number of errors, we do not report statistical analyses of accuracy or errors.

Figure 4 shows mean saccadic reaction times for correct trials in the RJA and RJAc

305

306

307

308

309

310

311

312

313

314

315

316

317

conditions. Participants were significantly slower on the Search task than the NoSearch task

(main effect of task $\left(F(1,15)=11.07, p=.005, \eta_{G}^{2}=0.13\right)$. They were also significantly slower to respond on RJA trials than RJAc trials overall (main effect of condition, $F(1,15)=98.75, p<$ $\left..001, \eta_{G}^{2}=0.57\right)$. Importantly, there was also a significant task ${ }^{*}$ condition interaction $(F(1,15)=$ 43.86, $\left.p<.001, \eta_{G}^{2}=0.18\right)$, indicating a larger effect of task in the RJA condition. This interaction was also present in all re-analyses of the data (see Supplementary Material 1). Follow-up paired t-tests revealed that responses to social gaze were significantly slower in the Search task than the NoSearch task $(t(15)=4.82, p<.001)$, whereas response times to arrow cues did not significantly differ between the two versions of the control task $(t(15)=-$ $0.85, p=.411)$. Consistent with our previous studies, there was an effect of condition (i.e., slower responses to gaze cues than arrow cues) for the Search $(t(15)=9.31, p<.001)$ task. However, there was also a significant (albeit smaller) effect of condition for the NoSearch task $(t(15)=8.51, p<.001)$ 


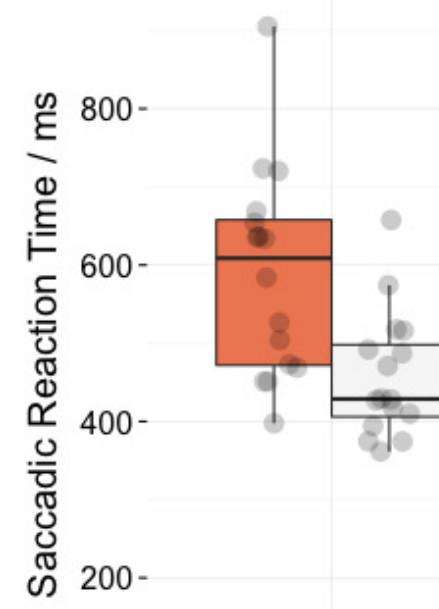

$0-$
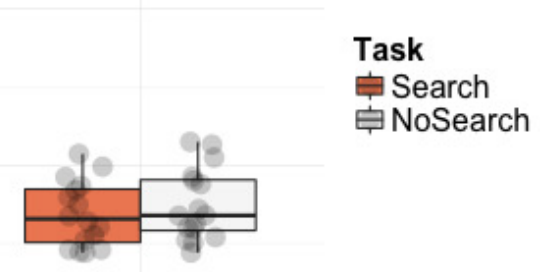

Figure 4. Box plots displaying saccadic reaction times in RJA and RJAc conditions, separated by task (i.e., Search, NoSearch). Data points represent individual participant means. 


\section{Discussion}

321 One of the main challenges facing social neuroscience - and the investigation of joint

322 attention in particular - is the need to achieve ecological validity whilst maintaining

323 experimental control. During genuine joint attention experiences, our social cognitive faculties

324 are engaged whilst we are immersed in complex interactions consisting of multiple social cues

325 with the potential for communication. A critical but neglected aspect of joint attention is the

326 requirement to identify those cues that are intended to be communicative. In the specific case of

327 eye gaze cues, the responder must differentiate gaze shifts that signal an intentional joint

328 attention bid from other, non-communicative gaze shifts. The results of the current study indicate

329 that this intention monitoring process has a measurable effect on responsive joint attention

330 behaviour.

331 The Search version of our Catch-the Burglar task was identical to that used in our

332 previous studies. In the social (RJA) condition, participants found all of their houses to be empty,

333 waited for their partner, Alan, to complete his search, make eye contact, and then guide them to

334 the burglar's location. We replicated our previous finding (Caruana et al., 2015; Caruana et al., in

335 press) that participants were slower to respond in this condition than in the matched control

336 (RJAc) condition in which the avatar's eye gaze cue was replaced by an arrow.

337 The critical innovation of the current study was the addition of a NoSearch condition in

338 which the same gaze and arrow cues were used but the joint attention episode was not preceded

339 by a search phase, thereby removing the intention monitoring component of the RJA condition.

340 Participants were still slower to respond to eye gaze than to arrow cues, suggesting that the

341 previously identified difference between RJA and RJAc in the Search condition is not entirely

342 attributable to intention monitoring. As discussed earlier, it is possible that differences in the 
343 perceptual salience of the arrow cue might help contribute this effect. Alternatively, participants

344 may be affected by the presence of a social partner. The current study was designed to control for

345 such factors and it is not possible to determine which if either of these explanations is correct.

346 The important finding was the task by condition interaction. This arose because the

347 magnitude of the condition effect was significantly reduced in the NoSearch version of the task.

348 This cannot be explained in terms of perceptual salience or social context, because these were

349 identical across Search and NoSearch tasks.

350 The interaction can also be viewed by contrasting the effect of task (Search vs.

351 NoSearch) for the two different conditions (RJA or RJAc). In the RJA condition, participants

352 were significantly faster to respond to the eye gaze cue when the search phase was removed. The

353 search phase required the participant and their virtual partner to make multiple non-

354 communicative eye-movements prior to the joint attention episode. Participants therefore had to

355 differentiate between eye-movements made by the avatar that signalled a communicative joint

356 attention bid and those that were merely a continuation of their search. In the NoSearch task,

357 every eye-movement made by the avatar was communicative, thereby removing the requirement

358 to monitor his communicative intent, enabling faster response times.

359 Importantly, there was no effect of task (Search vs. NoSearch) for the RJAc condition.

360 This allows us to discount a number of alternative explanations for the task effect in the RJA

361 condition. For example, it could be argued that the slower responses in the Search task reflected

362 differences in the timing of the stimulus presentation (e.g., the delay between participants

363 fixating on the avatar and the avatar making his guiding saccade). However, the timing of the

364 stimuli were programmed to be identical in the corresponding RJA and RJAc conditions of the

365 Search and NoSearch tasks, so any effect of stimulus timing should have been evident in both 
366 conditions. Another plausible explanation is that participants were slower in the Search task

367 because this required them to switch from searching for the burglar to responding to the avatar

368 on each trial. But again this applied equally to the RJA and RJAc conditions, so it cannot explain

369 the task by condition interaction.

370 In short, the observed interaction between task and condition is entirely consistent with

371 our intention monitoring account and cannot be explained in terms of the perceptual salience of

372 different cues, the task's social context, the timing of the stimulus presentation, or the

373 requirement to switch between searching and responding.

374 The current data provide insights into our other recent findings in studies using the

375 Search version of our interactive task. In one study, we used fMRI to investigate the neural

376 correlates of RJA (Caruana et al., 2015). By contrasting activation in the RJA (eye gaze) and

377 RJAc (arrow) conditions, we identified a broad frontotemporoparietal network including the

378 right temporo-parietal junction and right inferior frontal lobe. These brain regions are strongly

379 associated with aspects of social cognition including mentalising (e.g., Saxe \& Kanwisher, 2003)

380 and predicting anothers' actions (Danckert et al., 2002; Hamilton \& Grafton, 2008) but have not

381 been previously linked to RJA (cf. Redcay et al., 2012; Schilbach et al., 2010). The tasks used in

382 previous studies of RJA were similar to the current NoSearch task. As such, they would not have

383 captured the intention monitoring processes involved in RJA, perhaps explaining the

384 discrepenacy with our fMRI study. Future neuroimaging studies of joint attention could employ

385 the current study's design, and compare activation observed during the Search and NoSearch

386 task. If our interpretation is correct then removing the search component should reduce the

387 involvement of temporoparietal and inferior frontal regions in the RJA condition. 
In another study (Caruana, Stieglitz Ham, et al., in press), we investigated joint attention

389 in adults with autism. Observational studies of real-life interactions provide overwhelming

390 evidence that joint attention impairments are a core feature of autism (Charman et al., 1997;

391 Dawson et al., 2004; Loveland \& Landry, 1986; Mundy et al., 1990; Osterling, Dawson, \&

392 Munson, 2002; Wong \& Kasari, 2012). However, previous computer-based experimental studies

393 of joint attention have largely failed to find consistent evidence of gaze following difficulties

394 (Leekam, 2015; Nation \& Penny, 2008). One possible explanation for this is that autistic

395 individuals have an underlying difficulty in understanding the social significance or

396 communicative intentions conveyed by eye contact (cf. Böckler, Timmermans, Sebanz, Vogeley,

397 \& Schilbach, 2014; Senju \& Johnson, 2009) that is not captured by the tasks used in previous

398 studies of autism. In contrast to these studies, we did find evidence of impairment: autistic adults

399 made more errors and were slower to respond than control participants in the RJA condition

400 despite showing no impairment on the control condition. Future studies involving individuals

401 with autism and the Search and NoSearch versions of our task would clarify this issue further.

402

Another avenue for further research using this task would be to investigate the

403 development of joint attention in young children. Infants begin responding to and initiating joint

404 attention bids within the first year of life (Mundy et al., 2007), but virtual reality tasks provide

405 the sensitivity to investigate the developmental changes in the speed and efficiency of joint

406 attention engagement in later development. Finally, it would be of interest to investigate sex

407 differences in performance. Studies of infants (Saxon \& Reilly, 1999) and school-aged children

408 (Gavrilov, Rotem, Ofek \& Geva, 2012) have found that females exhibit increased joint attention

409 behaviours compared to their male peers, although it is unclear to what extent these differences

410 reflect underlying differences in competence as opposed to motivation. A limitation of the 
411 current study is that only three participants were female. However, future studies with larger

412 samples would allow systematic investigation of sex differences in joint attention performance at

413 multiple points across development.

414 Summary

415 In everyday joint attention episodes, a critical aspect of responding to joint attention bids

416 is the ability to discern which social cues have communicative intent and which do not. The

417 results of the current study indicate that this intention monitoring component has a measureable

418 effect on responding behaviour. Moreover, this component can be isolated by contrasting joint

419 attention episodes occurring in the context of a realistically complex social interaction versus a

420 simplified context in which each cue is unambiguously communicative. The clear differences in

421 performance on the Search and NoSearch versions of our task highlight the importance of

422 striving for ecological validity in studies of social cognition (cf. Schilbach et al., 2013). The

423 results also demonstrate the potential of our task for investigating the different components of

424 joint attention in typically developing children and in clinical populations associated with

425 atypical social cognition. 
427 Adamson, L. B., Bakeman, R., Deckner, D. F., \& Romski, M. A. (2009). Joint engagement and

428

429

430

431

432

433

434

435

436

437

438

439

440

441

442

443

444

445

446 the emergence of language in children with autism and Down syndrome. Journal of Autism and Developmental Disorders, 39(1), 84-96. doi: 10.1007/s10803-008-0601-7

Bayliss, A. P., di Pellegrino, G., \& Tipper, S. P. (2005). Sex differences in eye gaze and symbolic cueing of attention. Quarterly Journal of Experimental Psychology, 58, 1-20.

Bates DM. 2005. Fitting linear mixed models in R. R News 5:27-30

Böckler, A., Timmermans, B., Sebanz, N., Vogeley, K., \& Schilbach, L. (2014). Effects of observing eye contact on gaze following in high-functioning autism. Journal of Autism and Developmental Disorders, 44(7), 1651-1658. doi: 10.1007/s10803-014-2038-5

Bruinsma, Y., Koegel, R. L., \& Koegel, L. K. (2004). Joint attention and children with autism: A review of the literature. Mental Retardation \& Developmental Disabilities Research Reviews, 10(3), 169-175. doi: 10.1002/mrdd.20036

Bruner, J. S. (1974). From communication to language, A psychological perspective. Cognition, 3(3), 255-287. doi: 10.1016/0010-0277(74)90012-2

Bruner, J. S. (1995). From joint attention to the meeting of minds: an introduction. In C. Moore \& P. J. Dunham (Eds.), Joint attention: its origins and role in development. (pp. 1-14). Hillsdale, NJ: Lawrence Erlbaum Asscoiates.

Caruana, N., Brock, J., \& Woolgar, A. (2015). A frontotemporoparietal network common to initiating and responding to joint attention bids. NeuroImage, 108, 34-46. doi: 10.1016/j.neuroimage.2014.12.041 
447 Caruana, N., de Lissa, P., \& McArthur, G. (2016). Beliefs about human agency influence the 448 neural processing of gaze during joint attention. Social Neuroscience,

449 doi:10.1080/17470919.2016.1160953

450 Caruana, N., Stieglitz Ham., H., Brock, J., Woolgar, A., Palermo, R., Kloth, N., \& McArthur, G. 451 (in press). Joint Attention Difficulties in Adults with Autism. Autism.

452 Cary, M. S. (1978). The role of gaze in the initiation of conversation. Social Psychology, 41(3), 453 269-271. doi: 10.2307/3033565

454 Charman, T. (2003). Why is joint attention a pivotal skill in autism? Philosophical Transactions 455 Royal Society London Biological Sciences, 358, 315-324. doi: 10.1098/rstb.2002.1199

456 Charman, T., Swettenham, J., Baron-Cohen, S., Cox, A., Baird, G., \& Drew, A. (1997). Infants 457 with autism: An investigation of empathy, pretend play, joint attention, and imitation. Developmental Psychology, 33(5), 781-789. doi: 10.1037/0012-1649.33.5.781

459

460

461

462

463

464

465

466 467

468
Danckert J, Ferber S, Doherty T, Steinmetz H, Nicolle D, Goodale MA (2002) Selective, nonlateralized impairment of motor imagery following right parietal damage. Neurocase 8 , 194-204

Dawson, G., Toth, K., Abbott, R., Osterling, J., Munson, J. A., Estes, A., \& Liaw, J. (2004). Early social attention impairments in autism: Social orienting, joint attention, and attention to distress. Developmental Psychology, 40(2), 271-283. doi: http://dx.doi.org/10.1037/0012-1649.40.2.271

Frischen, A., Bayliss, A. P., \& Tipper, S. P. (2007). Gaze cueing of attention: visual attention, social cognition, and individual differences. Psychological Bulletin,133(4), 694-724. doi: $10.1037 / 0033-2909.133 .4 .694$ 
469 Gavrilov, Y., Rotem, S., Ofek, R., \& Geva, R. (2012). Socio-cultural effects on children's 470 initiation of joint attention. Frontiers in Human Neuroscience, 6, 286.

471 Hamilton AFC, de, Grafton ST. 2008. Action outcomes are represented in human inferior 472 frontoparietal cortex. Cerebral Cortex, 18 (5), 1160-1168.

473 Lawrence MA (2013). ez: Easy analysis and visualization of factorial experiments. R package 474 version 4.2-2, URL http://CRAN.R-project.org/package=ez.

475 Leekam, S. (2015). Social cognitive impairment and autism: what are we trying to explain?

476 Philosophical Transactions of the Royal Society of London B: Biological Sciences, 371,

477 1686.

478 Lord, C., Risi, S., Lambrecht, L., Cook, E.H., Leventhal, B.L., DiLavore, P.C., Pickles, A., 479 Rutter, M., 2000. The autism diagnostic observation schedule-generic: a standard 480 measure of social and communication deficits associated with the spectrum of autism.

481

482 Journal of Autism and Developmental Disorders, 30(3), 205-223. doi:

483

484

485

486

487

488

489

490

491

10.1023/A:1005592401947

Loveland, K. A., \& Landry, S. H. (1986). Joint attention and language in autism and developmental language delay. Journal of Autism and Developmental Disorders, 16(3), 335-349. doi: 10.1007/BF01531663

Mundy, P., Sigman, M., \& Kasari, C. (1990). A longitudinal study of joint attention and language development in autistic children. Journal of Autism and Developmental Disorders, 20(1), 115-128. doi: 10.1007/bf02206861

Mundy, P., Block, J., Vaughan Van Hecke, A., Delgado, C., Parlade, M., Pomeras, Y. (2007). Individual differences in the development of joint attention in infancy. Child Development, 78, 938-954. 
492 Murray, D. S., Creaghead, N. A., Manning-Courtney, P., Shear, P. K., Bean, J., \& Prendeville, J. 493 A. (2008). The relationship between joint attention and language in children with autism

494

495

496

497

498

499

500

501

502

503

504

505

506

507

508

509

510

511

512

513 spectrum disorders. Focus on Autism \& Other Developmental Disabilities, 23(1), 5-14. doi: $10.1177 / 1088357607311443$

Nation, K., \& Penny, S. (2008). Sensitivity to eye gaze in autism: Is it normal? Is it automatic? Is it social? Development and Psychopathology, 20(01), 79-97. doi: doi:10.1017/S0954579408000047

Oberwelland, E., Schilbach, L., Barisic, I., Krall, S. C., Vogeley, K., Fink, G. R., HerpertzDahlmann, B., Konrad, K., Schulte-Rüther, M. (2016). Look into my eyes: Investigating joint attention using interactive eye-tracking and fMRI in a developmental sample. NeuroImage, in press.

Osterling, J., Dawson, G., \& Munson, J. A. (2002). Early recognition of 1-year-old infants with autism spectrum disorder versus mental retardation. Development and Psychopathology, 14(02), 239-251. doi: doi:10.1017/S0954579402002031

Redcay, E., Dodell-Feder, D., Mavros, P. L., Kleiner, A. M., Pearrow, M. J., Triantafyllou, C., Gabrieli, J. D., \& Saxe, R. (2012). Atypical brain activation patterns during a face-to-face joint attention game in adults with autism spectrum disorder. Human Brain Mapping, 34, 2511-2523. doi: 10.1002/hbm.22086.

Saito, D. N., Tanabe, H. C., Izuma, K., Hayashi, M. J., Morito, Y., Komeda, H., . . Sadato, N. (2010). "Stay Tuned": inter-individual neural synchronization during mutual gaze and joint attention. Frontiers in Integrative Neuroscience, 4, 127. doi:

$10.3389 /$ fnint. 2010.00127 
514 Saxe, R., \& Kanwisher, N. (2003). People thinking about thinking people: The role of the

515 temporo-parietal junction in "theory of mind". NeuroImage, 19(4), 1835-1842. doi:

$516 \quad$ http://dx.doi.org/10.1016/S1053-8119(03)00230-1.

517 Saxon, Terrill F., and John T. Reilly. (1999). Joint Attention and Toddler Characteristics: Race, 518 Sex and Socioeconomic Status. Early Child Development and Care, 149(1), 59-69.

519 Schilbach, L., Wilms, M., Eickhoff, S. B., Romanzetti, S., Tepest, R., Bente, G., Shah N. J., 520 Fink, G. R., \& Vogeley, K. (2010). Minds made for sharing: Initiating joint attention 521 recruits reward-related neurocircuitry. Journal of Cognitive Neuroscience, 22(12), 2702$522 \quad$ 2715. doi: 10.1162/jocn.2009.21401.

523 Schilbach, L., Timmermans, B., Reddy, V., Costall, A., Bente, G., Schlicht, T., \& Vogeley, K. 524 (2013). Toward a second-person neuroscience. Behavioral and Brain Sciences, 36(4), 525 393-414. doi: 10.1017/S0140525X12000660.

526 Senju, A., \& Johnson, M. H. (2009). The eye contact effect: mechanisms and development.

527 Trends in Cognitive Sciences, 13(3), 127-134. doi:

528 http://dx.doi.org/10.1016/j.tics.2008.11.009

529 Singular Inversions. (2008). FaceGen Modeller (Version 3.3) [Computer Software]. Toronto, $530 \quad$ ON: Singular Inversions.

531 SR Research. (2004). Experiment Builder (Version 1.10.165). Ontario.

532 Stone, W. L., Ousley, O. Y., \& Littleford, C. D. (1997). Motor imitation in young children with 533 autism: what's the object? Journal of Abnormal Child Psychology, 25, 475-485. doi: 10.1023/A:1022685731726 
535 Tomasello, M. (1995). Joint attention as social cognition. In C. Moore \& P. J. Dunham (Eds.),

$536 \quad$ Joint Attention: Its Origins and Role in Development. Hillsdale: Lawrence Erlbaum

$537 \quad$ Associates.

538 Welch, B. L. (1947). The generalization of 'student's' problem when several different population $539 \quad$ variances are involved. Biometrika, 34, 28-35.

540 Wong, C., \& Kasari, C. (2012). Play and joint attention of children with autism in the preschool 541 special education classroom. Journal of Autism and Developmental Disorders, 42(10), $542 \quad$ 2152-2161. doi: $10.1007 / \mathrm{s} 10803-012-1467-2$

543 Wykowska, A., Wiese, E., Prosser, A., Müller, H. J., \& Hamed, S. B. (2014). Beliefs about the 544 minds of others influence how we process sensory information. PLoS ONE, 9(4), e94339. 545 doi:10.1371/journal.pone.0094339 